\title{
Síndrome cerebrohepatorrenal de Zellweger: una enfermedad peroxisomal
}

\author{
Eliana Rodillo B. '; Marcos Vallejos A.'; \\ León Adlerstein S. '; Wanda Fernández. M. ${ }^{2}$; Sergio Gonź́lez B.+; \\ María E. Kawada S., ${ }^{5}$ Manuel J. Santos A.4
}

\section{Zellweger syndrome: a peroxisomal abnormality}

An ritent with craneofacio cysmorphia, hepatonegaly, kidney cysts and severe neurobgical dysfunction is repoted. No ab-ormalifies were detected on either serun: orrinoxcid, lactic acid and ammonic levels or urinory aninoacid excretion, but accumulation of very ons chain faly ac ds abnormal subcellular distribution of the peroxisonal enzyme catalose cnd seroxisomal grosts were found in cultured fibreblasts. These clinical and laboratory fincincs supoart the diognosis of Zellweger (cerebrohepasondi syndrone, o peroxisorral disease.

'Key words: Ze liweger syndrome, cerebrohiepatorenol syndrore.'

El síndrome cerebrohepatorrenal o síndrome de Zellweger, es una cnfermedad autosómica recesiva. Ictal, cuya incidencia es estimada entre 2 y 4 por 100000 nacidos vivos ${ }^{1}$. Corresponde al prototipo de las llamadas enformedades peroxisomales 2.3 . Se caracteriza clínicamente por dismorfias craneofaciales, hepatomegalia y disfunción neurológica severa'. En esta presentación se describen las características clínicas y de laboralorio del primer paciente con sindrome de Zellweger identificado en nuestro país y se revisa el tema con el propósito de estimular la búsqueda y contribuir al estudio de estos raros pacientes.

1. Servicso de Neurología, Hospital Roberto del Río.

2. Servicio de Analomía Patológica, Hospital Roberto del Río.

3. Departamento Anaromía Patológica. Faculiades de Ciencias Biológicas y Medicina, Pontifician Universidad Católica de Chile.

4. Departamentos de Biología Celular y Molecular y Pediatría. Faculsades de Ciencias Biológicas y Medicina. Pontificia Uniyersidad Catolica de Chile.

5. Técnóloga Médica. Departamentos de Biología Celelas y Molecular y Pediatria, Facultades de Ciencias Bioló gicas y Medicina. Pontificia Universidad Catolica de Chile.

Financiado parcialmente por Fondecyt 1940686

\section{Caso clínico}

Recién nacido masculino, de término. Al nacer pesaba $2900 \mathrm{~g}$. Calificación Apgar 9 al minulo, 9 a los 5 minutos. Síndronse de dificultad respiratoria, bronconeumonía a hipoglicemia asintomftica en período postnalad inmediato. Posteriormente sufrió bronconeumonía intrahospitalaria que se trató con ampicilina y amikacina. Duraute su hospitalización se registró hipotonía severa, dismorfias faciales y pie bot bilateral, para lo cual se indić yeso corrector en otro hospital. Fue referido a nuestro servicio para completar su estudio. Ingresó con dificultad respiratoria, hipoIonía severa y retención de $\mathrm{CO}_{2}$, por lo que fue conectado a ventilación necárica. Su bregna era amplio, las suturas del críneo separadas. frente amplia, epicanto, paladar ojival, cuello corto y hepatomegalia. No se conectaba bien con el medio. Hipactivo. Reflejo de Muro y succión auseftes. Hipotonía extrema. Durante su hospttalización sufrió convulsiones que respondieron favorablemente a fenobarbital. Por la asociación de dismorfias faciales, hiporonía severa, hepatomegalia y convulsiones se sospecho el síndrome de Zellwèger. La ecografía cerebral mostró lesiones quísticas en ambas cabezas de los núcleos caudados. En la tomograffa axial cerebral se registró hipodensidad de la sustancia blanca en la región frontoparietal derecha $y$, en la ecografía abdominal, un quiste renal y hepotomegalia. La biopsja muscular y la actividad sérica de CPK (crealina fosfoquinasa) eran normales. En el examen ocular se encontró restos de membrana mesodérmica en el itis de amibos ojos. pequeña opacidad del limbo corneal derecho $y$ papilas pạlidas. El rastreo metabólico para aminoacidemia, aminoaciduria ácido láctico y amonio dio resultados normales. Los ácidos grasos de cadena muy larga estaban muy aumentados en el plastna, especialnente en C26:0 = 6.289 
$\mu \mathrm{g} / \mathrm{ml}$ en plasma (normal aproximadamente $0,33 \pm 0,18$ $\mu \mathrm{g} / \mathrm{ml}$ ). La distribución subcelular de la catalasa peroxisomal en cultivo de fibroblastos, mediante fraccionamiento celular. mosıró que $\mathbf{9 0 \%}$ de la enzima estaba libre en el citoplasma y la técnica de immunodetección de proteinas peroxisomales en fibroblastos de piel y en la biopsia de bigado revelo gran escasez de peroxisomas (visualizados como partículas que contienen catalasa) y su reemplazo por sacos membranosos peroxisomales vacios (fantasmas peroxisonales). Cabe senalar que en cultivos de fibroblastos se detectaron algunos peroxisomas residuales mediante inmunofluorescencia indirecta con anticuerpos anti catalasa (figura). Con estos antecedentes se formulo el diagnóstico de síndrome de Zellweger. A los seis meses de edad el paciente fue trasladado a su ciudad de origen, donde permaneció hospitalizado, sin mostrar avances en su desarrollo psicomotor y suftiendo repetidas bronconeumorías, hasta la edad de 10 meses de edad, en que falleció, al parecer a causa de una de ellas.

\section{Comentario}

El diagnóstico de sindrome de Zellweger debe sospecharse clínicamente en todo niño con
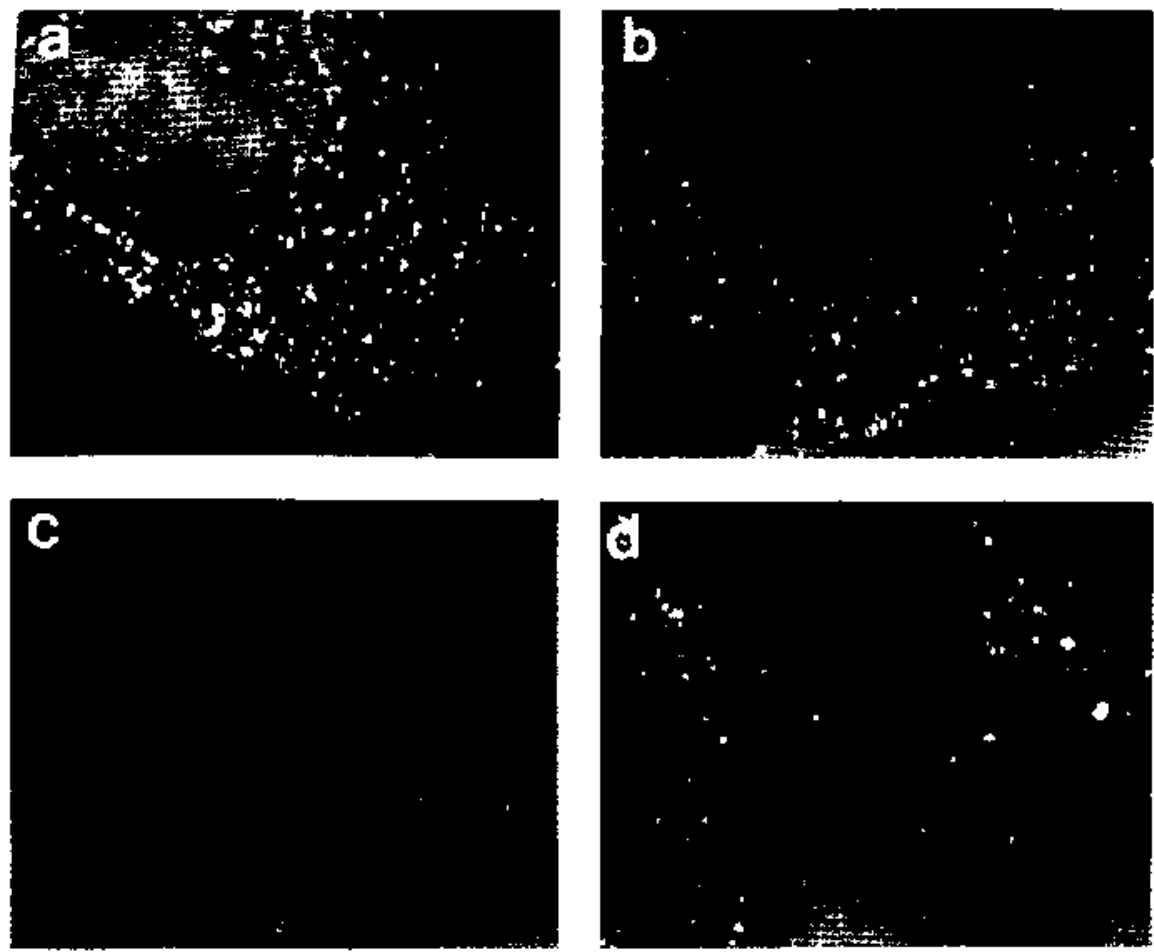

Figura: Inmunodetección de peroxisemas en fibroblastos en cultivo, mediante inmunofluores. cencia indirecta. Fibroblastos en cultivo de un sujeto control (a y b) y del paciente (c y d), fueron cultivados y sometidos a inmunofluorescencia indirecta. utilizando anticuerpos anticatalasa (a y c) y antiproteínas de membrana peroxisomal (b y d). En fibmoblastos controles se observan abundantes peroxisomas normales ( $a$ y b). en tanto en los fibroblastos del paciente se observan abundantes fantasmas peroxisomales (d) y muy escasos peroxisomas residuales (c), (objetivo $100 \%$ ). 
oxidación de ácidos grasos de cadena muy larga (>) 22 átomos de carbono), resultando en un acortamiento de éstos a cadenas medianas y cortas (6-8 átomos de carbono); la síntesis de éter-fosfolipidos, como los plasmalógenos; la oxidación de varios ácidos como el fítánico y dicarboxilicos ${ }^{4}$. Las proteínas (enzimas) peroxisomales son sintetizadas en los polirribosomas del citoplasma y son importadas al organelo en forma postraduccional. de modo que el organeto crece y se divide por fisión. En la membrana peroxisomal existe una maquinaria de importación encargada de reconocer (receptores) y transportar (translocasas dependiente de ATP) las proteínas peroxisomales. Si existe una falla en este mecanismo de importacion. las proteínas peroxisomales no son capaces de incorporarse a los peroxisomas, quedando libres en el citosol donde pueden permanecer activas o ser degradadas ${ }^{5}$.

Las enfermedades peroxisomales se dividen en tres grupos (tabla) ${ }^{3}$ : En el grupo I los peroxisomas están muy disminuidos y hay deficiencia de múltiples funciones peroxisomales. Su prototipo corresponde al síndrome de Zellweger, e incluyen a otras afecciones tales como: adrenoleucodistrofia neonatal (NALD), enfermedad de Refsum infantil (IRD) y acidemin hiperpipecolica (HA). Todas estas enfermedades, a su vez, se consideran afecciones de la biogénesis peroxisomal ${ }^{5}$. En el grupo Il los peroxisomas están presentes y existe más de una función peroxisomal alterada, como ocurre con la condrodisplasia punctata de tipo rizomélico (RCDF) y el síndrome tipo Zellweger y en el grupo III los peroxisomas están presentes, pero hay pérdidas de una función (enzima) peroxisomal. Integran este grupo las típicas afecciones genéticas metabólicas debido a fallas de una enzima peroxisomal. La más conocida es la adrenoleucodistrofia ligada al cromosoma $X$, en que habría una falla de la enzima que activa a los ácidos grasos (ligasa) para su posterior oxidación ${ }^{3}$.

Las manifestaciones clínicas del síndrome de Zellweger fueron descritas por Bowen en $1964^{\circ}$. Los pacientes afectados son letárgicos, inactivos

\section{Tabla}

Clasificación de las enfermedades peroxisomales

\section{Grupa I}

Peroxisonas deficientes y pérdida generalizada de funciones peroxisomates (afecciones de la biogénesis peroxisomal)

Síndrone cerebrohepatorrenal (Zellweger) (ZS)

Adrenoleucodisirofia nconatal (VALD)

Entermedad de Refsum infantil (IRD)

Acidemia hiperpipecólica (HPA)

\section{Grupo II}

Peroxisomas presentes y pérdida de múltiples funciones peroxisomales

Condrodisplasia punitata tipo nzomélico (RCDP)

Sindrome tipo Zallweger

\section{Grupo III}

Peroxisomas presentes y pérdida de una función peroxisomal

Adrenoleukodistrofia ligada al $X(X-A L D)$ y sus variantes

Defocjencja del sislema de B-oxidacion peroxisomal:

Deficiencia de atit-CoA oxidasa (psetrdo-NALD)

Deficiencia de proteína bifuncional

Deticiencia de tiolasa peroxisomal (pseudo-ZS)

Deficiencia de dehidroxiacetona fosfato aciltransferasa (deficiencia de DHAPAT. pseudo-RCDP)

Deficiencia de glutaril-CoA oxidasa

Acidemia Di-y trihydroxicolestanoica

Hiperoxaluria tipo I

Acilalasenia 
y sufren convulsiones. No responden a estimulos externos, succionan o degluten. Muestran hipotonia severa, arreflexia y no tienen reflejo de Moro. La hipotonía es de origen supranuclear, pero se asemeja a la neuromuscular. Estos pacientes presentan dismorfias craneofaciales como frente alta, suturas frontales muy amplias, reborde supraorbitario plano, implantación baja de las orejas y paladar alto. Las alteraciones oculares incluyen telecanto, opacidades corneales, cataratas, glaucoma, alteraciones retinianas y displasia del nervio óptico. Existe hepatomegalia y alteración de las pruebas hepáticas, quistes rcnales y detención del desarrollo psicomotor. Su expectativa de vida es muy baja: $70 \%$ fallecen en los primeros seis meses de vida y $92 \%$ en cl primer año. La sobrevida más allá de los 2 años y medio de vida es excepcional!

En 1973 Goldfischer y cols. describieron la ausencia de peroxisomas en hígado de pacientes con sindrome de Zellweger?. Posterionmente, se demostro que en otros pacientes la enzima catalasa (cl marcador biológico para los peroxisomas) se encontraba libre en el citosol ${ }^{\mathrm{x}}$. Otras enzimas, como el sistema de B-oxidación peroxisomal estaban ausentes. Santos y cols en $1988^{10}$ usando técnicas de inmunodetección de proteínas de la membrana peroxisomal, encontraron que cstas membranas estaban presentes en forma íntegra. pero su interior estaba vacío (ausencia de proteînas y enzimas de la matriz) denominando a estas estructuras como "fantasmas peroxisomales". El defecto genético de esta afección podría residit a nivel de la maquinaria de importación de proteínas al interior del peroxisoma. ejemplificando un nuevo tipo de error congénito del metabolisino: una afección organelar, que afecta la biogénesis de todo un organelolo. Hay gran heterogeneidad genética entre los pacientes con síndrome de Zellweger, mencionándose alrededor de 10 grupos de complementación genética distintos ${ }^{11}$, en tres de los cuales se han identificado las fallas, que corresponden a alteraciones de proteinas de la membrana peroxisomal, presumiblemente involucradas en la importación ${ }^{11}$.

Los hallazgos neuropatológicos en el sindrome comprenden anormalidades en la citoarquitectura cerebral, como nicrogiria y paquigiria, heterotopias y desmielinización 12. 12. Ellas se han atribuido a la deficiencia de enzimas peroxisomales. Así, en animales de experimentación el déficit de ácido pipecólico produce hipotonía'. Los plasmalógenos son importantes constituyentes de la membrana celular eléctricamente activa en el cerebro, representando 80 a $90 \%$ de los fosfolípidos de la sustancia blanca cerebral. Los peroxisomas son muy numerosos en las células especializadas en el metabolismo lipídico, tales como los oligodendrocitos, que producen la mielina del sistema nervioso central. En ellos los peroxisomas aumentan concordantemente con la máxima producción de mielina, sugiriendo un rol importante en la mielogénesis ${ }^{13}$. En fetos con síndrome de Zellweger los defectos de migración se inician alrededor del tercer mes de gestación y han sido relacionados con la acumulación de ácidos grasos de cadena muy largat.

El estudio de laboratorio del síndrome de Zellweger y de las enfermedades peroxisomales debiera incluir, entre otros, la detenminación de la concentración de los ácidos grasos de cadena muy larga en sangre (o tejidos), la evaluación de la distribución subcelular de enzimas peroxisomales (p.e. catalasa) en fibroblastos en cultivo o hepatocitos (enzima que en la enfermedad Zellweger se encuentra en su mayoría libre en el citosol), la cualuación de la ausencia de peroxisomas normales y su reemplazo por los fantasmas peroxisomales, mediante inmunodetección de enzimas de la matriz y proteínas de la membrana peroxisomal en fibroblastos en cultivo o biopsia hepática, la medición del ácido fitánico y de ácidos biliares; la determinación de la actividad de las enzimas peroxisomales de la biosíntesis de plasmalógenos ${ }^{3,5}$. Recientemente, también se ha utilizado linfoblastes (linfocitos transformados por el virus Epstein Barr) para estudios del sindrome de Zellweger ${ }^{14}$. Nuestro paciente presentaba, además de los signos clínicos característicos, aumento de ácidos grasos de cadena muy larga (C26:0), presencia de catalasa mayoritariamente en el citoplasma, y fantasmas peroxisomales en fibroblastos y hepatocitos. Todos estos hallazgos fundamentan el diagnóstico de síndrome de Zellweger. Conviene señalar que un paciente con un fenotipo similar al reportado aquí, es decir con algunos peroxisomas residuales en sus fibroblastos, ya se había detectado anteriormente ${ }^{15}$.

Por último, cabe destacar que el diagnóstico prenatal es posible a través de la detección de ácidos de cadena muy larga en el cultivo de 
células de líquido amniótico ${ }^{16}$. El tratamiento para la enfermedad de Zellweger hasta el momento es sólo de apoyo'.

\section{Resumen}

Se describe el caso clínico de un lactante con dismorfias craneofaciales, hepatomegalia, quistes renales y disfunción neurológica severa. Los exámenes de rastreo para aminoacidemia, aminoaciduria, ácido láctico y amonio dicron resultados normales, pero había altas concentraciones plasmáticas de ácidos grasos de cadena muy larga, distribución subcelular anormal de la catalasa peroxisomal y fantasmas peroxisomales en fibroblastos cultivados. Estas características clínicas y de laboratorio sustentan el diagnóstico de sindrome de Zellweger.

(Palabras clave: síndrome de Zellweger. sindrome cerebrohepatorrenal.)

\section{Referencias}

1. Zellueger $H$; The ccrebro-hepalo-renal (Zellweger) syndrome and other peroxisomal disorders. Review article. Dey Med Child Neurol 1987: 821-829

2. Silutgeus $R$. Heimans $H$, Wanders $R$, el al.: Peroascomal Jisorders; a newly recognized group of genetic diseasises. Eur J Pediatr 1986: 144: 430-440.

3. Whilers $R$, Barh $P$. Schutgess $R$, el al. Clinical and biochemical characteristics of peroxisomal disorders: update. Eur 3 Pediats 1994: 1.53 (Supl.1): 544-548.

4. Move $H$ : The peroxisome: nervous system role of a previosly underrated organelle. Neurology 1988; 38 : 1617.1627
5. Luzarah PB. Moser H: Disorders of peroxisomal biogenesis. In: The metabolic basis of inherited diseases. Scriver C, Beaudet A, Sly W, Valle D (Eds.). McGraw Hill Co. New York, 6th Ed. 1989:1479-1509.

6. Bouten P. Lee C. Zellueger H. Lidenberg' R: A familiar syndrome of multiple congenital defects. Bull Johns Hopkins Hospital 1964; I14: 402-414.

7. Goldfischer S. Moure C. Johnson A, et al: Peroxisomal and mitocbondrial defects in the cerebro-hepato-renal syndrome. Science 1973: 182: 62-64.

B. Santor M. Ojeda J, Garrido d. et al: Peroxisamal organization in normal and cerebrohepatortenal (Zellweger) syndrome fibroblasts. Proc Naal Acad Sci USA 1985; 82: 6556-6560

9. Schrom AW, Strijlatd A. Hashinoto $T$, et at: Biosynthesis and maturation of peroxisomal b-oxidation enzymes in fibroblasts in relation to the Zellweger syndrome and infantile Refsum discase. Proc Natl Acad Sci USA 1986: 83: 6156.6158.

10. Santos MJ, Imanaka $T$, Shio $H$, et at: Peroxisomal membrane ghosts in Zellweger syndrome, aberrant organelle assembiy. Science 1988; 239: 1536-1538.

11. Dodt $G$. Braverman N. Wong $C$. et al: Mutations in the PTS $]$ receptor gene, PXR], define complementation group 2 of the peroxisome biogenesis disorders. Nature Genetics 1995; 9: 11.5-125.

12. Wanders R. Heymans $H$. Schutgens $R$, et al. Peroxisomal disorders in neurology. Review article. J Neurol Sci 1988: 8B: ]-39.

13. Brolly FR, Viright $R$. Singh AK. Singh f. Petoxisomal disorders neurodevelopmental and biochemical aspects. Review. AJDC 1993; 147:617-626.

14. Santas $M J$. Moser $A B$. Drwinga $H$, et al: Analysis of peroximes in lymphoblasts: Zellweger syndrome and a patient with a deletion in chromosome 7. Pediart Res 1993; 33: 441-444.

15. Sinter MJ. Hefefter S. Moser AB, et al: Peroxisomal asscmbly mutations in humans: structural heterogeneity in Zellweger syndrome. J Cell Physiol 1992; I51: I03. 112.

16. Moser AE. Singh AB, Brown FR, et af: Increased eveds $\&$ inpuired degradation of VLCA and their use in prematnl diagnosis. New Engl J Med 1984; 310; J I4 J-1 146.

\section{AVISO A LOS AUTORES}

Con el objeto de dar prioridad a los trabajos de investigación, en vista de las limitaciones de espacio de la Revista Chilena de Pediatría, el Comité Editorial ha acordado restringir la impresión de casos clínicos a un máximo de dos por cada número. 